Revue internationale d'éthique sociétale et gouvernementale

vol. 18, $n^{\circ} 2 \mid 2016$

Éthique et scandales publics

\title{
L'indignation est-elle un ressort de la scandalisation? Le « scandale des fiches » en Suisse
}

Hervé Rayner, Fabien Thétaz et Bernard Voutat

revues.org

Édition électronique

URL : http://

ethiquepublique.revues.org/2847

ISSN : 1929-7017

\author{
Éditeur \\ Éditions Nota bene \\ Édition imprimée \\ Date de publication : 1 septembre 2016 \\ ISSN : $1488-0946$
}

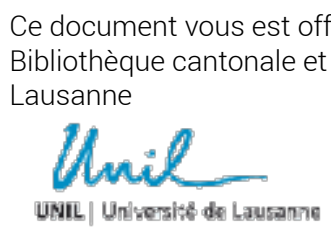

Référence électronique

Hervé Rayner, Fabien Thétaz et Bernard Voutat, « L'indignation est-elle un ressort de la

scandalisation? Le «scandale des fiches » en Suisse », Éthique publique [En ligne], vol. 18, n² 2 |2016, mis en ligne le 26 janvier 2017, consulté le 01 février 2017. URL : http://

ethiquepublique.revues.org/2847

Ce document a été généré automatiquement le 1 février 2017.

Tous droits réservés 


\title{
L'indignation est-elle un ressort de la scandalisation? Le « scandale des fiches » en Suisse
}

\author{
Hervé Rayner, Fabien Thétaz et Bernard Voutat
}

1 Événement récurrent dans les sociétés contemporaines, le scandale n'en constitue pas moins un objet mal identifié par les sciences sociales. Longtemps considéré comme un épiphénomène, fuyant, éphémère - voire indigne - difficilement observable, inclassable par rapport à des situations supposées mieux balisées ou structurantes (révolutions, crises politiques), il a d'abord été étudié dans une approche anthropologique et/ou fonctionnaliste d'inspiration durkheimienne. Or cette littérature s'intéresse moins au scandale en soi qu'à ce qu'il permet de révéler. À l'image de la relation entre le symptôme et la maladie, le scandale n'est pas étudié pour lui-même, mais pour ce qu'il éclaire de réalités considérées comme plus fondamentales (structures, institutions, cultures, normes, etc.). Ainsi, dans une perspective anthropologique déjà ancienne, proche de la première sociologie des religions (Durkheim, 1912), la réprobation morale constitutive du scandale est étudiée comme un rite expiatoire qui, par ses propriétés cathartiques, assurerait une fonction d'intégration sociale en rappelant à la conscience collective la validité de la norme. Dans un article précurseur, Éric de Dampierre voit dans le scandale un « test » par lequel une société remet en question son adhésion à des valeurs communes et dont la fonction est précisément de les renforcer, "par le fait même qu'il les sape » (1954: 335). Dans une même veine durkheimienne, Jeffrey Alexander interprète le scandale du Watergate comme un rite de revitalisation de la "conscience collective américaine », suivant un cycle «sacralisation, pollution, purification» (1988: 199), une approche que l'on trouve également chez Pierre Bourdieu lorsqu'il écrit: «Ainsi, le scandale politique, comme révélation, par la presse, d'une transgression éthique accomplie par une personnalité éminente, rappelle à la règle du dévouement à l'intérêt général » (1997: 148). 
2 Pour légitimes que soient ces perspectives d'analyse, elles tendent à inscrire le scandale dans une histoire naturelle "transgression - scandale - renforcement des valeurs communes" (scandal is corruption revealed, Lowi, 1988: 7), de sorte que l'approche fonctionnaliste induit une confusion entre le fait présumé scandaleux (la transgression) et le scandale (les mobilisations autour d'une dénonciation) et s'interdit par là même d'en penser l'occurrence aussi bien que la dynamique. Or toute transgression ne donne pas forcément lieu à un scandale; elle n'est pas une condition suffisante ni même nécessaire. $\underline{\text { Si scandale il y a, celui-ci ne débouche pas forcément sur une réaffirmation des valeurs }}$ centrales du système politique ou sur un renforcement des mécanismes de contrôle, comme le montre par exemple Camille Hamidi (2009) lorsqu'elle analyse le processus de dilution de la dénonciation médiatique dans l'affaire Pechiney. Même lorsque c'est le cas, il ne s'agit pas d'un produit «naturel» du scandale, comme le note par exemple John B. Thompson $(2000: 237)$ à propos du Watergate, mais le résultat en partie contingent des affrontements constitutifs du scandale.

3 À partir des années 1990, la littérature spécialisée prend une nouvelle orientation : non seulement le scandale devient un objet à part entière des sciences sociales, mais il est dorénavant théorisé comme une forme de mobilisation, irréductible aux comportements déviants censés être à son origine. Ainsi, Lawrence Shermann note que «les scandales sont des phénomènes socialement construits impliquant la coopération et le conflit entre de nombreuses personnes " $(1989: 887)$. Dans un article programmatique, Justin Daniel affirme que le scandale «tire sa consistance de la mobilisation d'un nombre restreint d'acteurs directement engagés dans la lutte pour le pouvoir et sollicitant l'attention d'un large public, via les moyens d'information» (1992: 985). Aujourd'hui, la plupart des travaux admettent l'absence de relation mécanique entre transgression et scandale. Cette approche «non spontanéiste » du scandale - on pourrait dire «constructiviste » - suit toutefois des perspectives assez différentes, certains le considérant comme une forme de lutte politique, d'autres insistant sur le rôle central des médias ou sur les justifications des protagonistes.

4 Représentant de l'approche stratégiste, Alain Garrigou rapporte l'émergence de scandales aux coups portés par des acteurs politiques «motivés par des calculs rationnels sur l'opportunité du dévoilement» (1992 : 27) afin de discréditer leurs adversaires. Rompant radicalement avec les approches en termes de moralisation, l'auteur adopte une conception "réaliste» du jeu politique, où le dévoilement de transgressions est instrumentalisé dans la lutte pour le pouvoir (Garrigou, 1993). On peut reprocher à cette vision stratégiste du scandale son caractère politico-centré (Rayner, 2005 : 12), les acteurs politiques n'étant pas les seuls capables de calculer. D'autre part, un scandale politique ne peut prendre qu'avec la "complicité » des médias, qui ne se bornent pas à relayer les échanges de coups politiques, mais participent directement à la définition de la situation. C'est pour l'essentiel la thèse de Thompson (2000), qui situe dans l'avènement de la presse à grand tirage au XIX ${ }^{e}$ siècle la condition nécessaire à l'émergence de la forme moderne du scandale, ce qui l'amène à souligner l'importance des médias comme l'arène où se publicisent les dévoilements de transgressions et leurs réprobations, où se font et se défont les capitaux symboliques des acteurs politiques. Pour leur part, Patrick Champagne et Dominique Marchetti assimilent l'affaire du sang contaminé à un "symptôme" des transformations du champ journalistique français, sous l'emprise croissante de l'information télévisée. Selon eux, «il est sans doute à peine exagéré de dire qu'est scandaleux ce que le champ journalistique, dans son ensemble, considère comme 
tel et parvient surtout à imposer à tous » (1994: 43). Cette perspective, à la manière du point de vue stratégiste, restreint l'attention à une seule catégorie d'acteurs réputés "faiseurs de scandale». En fait, tout scandale politique d'ampleur implique la participation d'acteurs situés dans différents espaces sociaux: élus, journalistes, intellectuels, magistrats, chercheurs, dirigeants associatifs, citoyens ordinaires.

La perspective ouverte par la sociologie pragmatiste (de Blic et Lemieux, 2005) opère un déplacement du questionnement en proposant de penser la dimension instituante du scandale, de saisir celui-ci comme un « moment de transformation sociale » possédant sa propre dynamique, un «test » ou une " épreuve ». Il s'agit alors moins de rendre compte de ce que le scandale pourrait "révéler » que d'analyser " ce qu'il fait » aux rapports sociaux. Écartant le questionnement étiologique, cette approche invite à focaliser l'attention sur les réactions sociales suscitées par une dénonciation publique, attendu que «c'est aux acteurs eux-mêmes, et à eux seuls, qu'il reviendra de nous montrer, à travers leurs réactions indignées ou leurs attitudes de relativisation, si la faute en est une » (de Blic et Lemieux, 2005, §13). Si nous sommes d'accord pour déplacer le regard sur la dynamique du scandale plutôt que sur ses causes et pour la penser dans son indétermination fondamentale comme un processus ouvert, à l'issue incertaine, il reste que l'approche pragmatiste reste captive d'une perspective rapportant les conduites à la dimension normative de l'agir. C'est ainsi que le scandale en vient à être défini, selon un raisonnement proche de Dampierre qui est parfaitement assumé, comme ce «test à travers lequel se manifeste le degré actuel d'adhésion d'une communauté à certaines normes " (de Blic et Lemieux, 2005, § 13). À leurs yeux, contrairement aux approches constructivistes et stratégistes, les démarches fonctionnalistes présentent l'intérêt de " [prendre] en considération l'existence de normes partagées [et] mettent en lumière le caractère non arbitraire - et même socialement obligatoire - de l'indignation suscitée par le scandale [...] » (de Blic et Lemieux, 2005, § 23). Fidèle à la sociologie des épreuves qui interroge les conditions de recevabilité d'une dénonciation publique d'injustice (Boltanski, 1990 : 20), il s'agit de "prendre au sérieux » les justifications et les critiques des acteurs aux fins d'en « explorer les effets » (Barthe et al., 2013 : 186), de mieux saisir, " contre le réductionnisme stratégiste », la réflexivité des acteurs, ainsi que «les raisons qu'a le public de se scandaliser ou de ne pas le faire » (de Blic et Lemieux, 2005 : § 23). Il s'agit en somme de " prendre la mesure [que] tout n'est pas construit dans un scandale [et que] si construction il y a [...] elle ne repose pas sur rien, mais sur des valeurs partagées " ou encore une " grammaire politique " plus ou moins commune (de Blic et Lemieux, 2005, §23). En définitive, «l'insistance sur l'agir régulé par des normes (ainsi que le dénomme Habermas) permet au chercheur de rendre un compte plus exact de l'aspect émotionnel et irréfléchi de l'indignation soulevée par le scandale [...]» (de Blic et Lemieux, 2005, $\S 24)$. On le voit, l'approche pragmatiste de Damien de Blic et Cyril Lemieux, en dépit de toutes les nuances apportées dans la discussion critique de la littérature, rapporte le scandale à la transgression de normes qui, une fois dénoncée en bonne et due forme, attiserait l'indignation. Celle-ci fait alors figure de point nodal dans l'approche pragmatiste du scandale, comme l'atteste l'ouvrage collectif que ses principaux représentants lui consacrent, qui s'organise autour de ces quatre parties : «l'indignation qui rassemble », « l'indignation qui divise », « l'indignation qui trouble », « l'indignation, objet des sciences sociales » (Boltanski et al., 2007). Si de Blic et Lemieux nous invitent aussi à " prendre toute la mesure de l'ancrage situationnel des acteurs » (2005: 27), celuici désigne des "régimes d'action ", à savoir des opérations de justification reposant sur des ordres argumentatifs que Boltanski et Thévenot appellent des « cités » par référence 
aux «sphères de justice » identifiées par Michael Walzer. S'agissant des scandales, les deux sociologues pragmatistes les étudient en termes de tension entre la "cité civique » et la «cité domestique» (Boltanski et Thévenot, 1991: 22). En définitive, parce qu'elle s'intéresse d'abord à la dimension normative des scandales et à leurs effets, la sociologie pragmatiste est peu à même de rendre compte de quoi ils sont faits (Rayner, 2015), question du reste qui ne figure pas à son programme de recherche, qui s'interdit par principe d'expliquer les conduites des acteurs en dehors des justifications qu'ils produisent.

6 Selon nous, c'est moins le contenu des normes (régimes d'action, cités ou grammaires) qui prime dans l'expression publique d'une indignation que la perception qu'ont les acteurs de la jouabilité de cette expression publique, c'est-à-dire de ce qu'ils estiment dicible et faisable ou non (avec toutes les gradations possibles entre ces deux bornes) dans la situation dans laquelle ils sont pris. Cette dimension est centrale pour saisir la dynamique des scandales. Elle constitue en quelque sorte le chaînon manquant entre les "grammaires normatives" (pour peu que l'on retienne cette notion) et les émotions, considérant "la relative stabilité des premières et la très forte volatilité des secondes " (Rayner et Thetaz, 2015: 182), un constat qui vaut aussi pour les mobilisations politiques et les "raisons d'agir» des individus qui s'y engagent. Cette perspective générale implique de mettre en œuvre un point de vue à la fois relationnel et processuel.

7 Tout d'abord, la dénonciation indignée d'une transgression, réelle ou supposée, de normes dépend des évaluations et des anticipations réciproques que les acteurs nourrissent à propos de la situation et de ce qu'elle rend possible pour eux-mêmes et pour les autres. En d'autres termes, si l'estimation par un individu de la jouabilité d'une dénonciation tend à le persuader ou à le dissuader d'exprimer son indignation, il en va de même pour ses interlocuteurs, car chacun évalue et agit en fonction de ce qu'il perçoit de ce que font les autres (Rayner, 2005). Immergées dans l'action, les perceptions du possible s'avèrent pour le moins contraignantes pour les individus. Elles disposent d'une force sociale au sens durkheimien, qui doit être pensée en relation avec les autres dimensions contraignantes des univers sociaux (champs, institutions) dans lesquels évoluent les acteurs, mais aussi en fonction de variables situationnelles hic et nunc. Le point de vue adopté ici invite à « comprendre la façon dont les participants à l'action collective - et les autres - donnent du sens à leur action, interprètent les situations auxquelles ils sont confrontés, perçoivent le possible et définissent le probable, anticipent les effets de leurs propres actes ou les coups joués par d'autres » (Dobry, 1993 : 182). Dans cette perspective, le scandale ne peut être lu comme mettant en jeu des valeurs ou significations que l'on supposerait communes à l'ensemble de ceux qui participent à l'action collective. L'indignation exprimée fait au contraire intervenir des acteurs pris dans des jeux de miroir, dont les « raisons » ou " motifs » sont hétérogènes, de même que leur capacité à agir est inégale, qui «se saisissent d'actions lancées par d'autres » pour les investir de « significations différentes à partir de lieux sociaux eux aussi différents » (Dobry, 1993 : 182). On comprend dès lors que ces perceptions du possible soient mouvantes, qu'elles peuvent évoluer très rapidement au gré d'ajustements en série (ripostes, échanges de coups ou ruptures des soutiens) et d'alignements collectifs peu prévisibles de la part des acteurs. C'est pourquoi l'analyse doit être attentive à la diachronie, à la dynamique ouverte et en partie contingente du scandale. À partir des mêmes structures sociales, les mobilisations à la base du scandale des fiches auraient pu ne pas avoir lieu. 
8 En définitive, un scandale ne prend place que si des acteurs mettant publiquement en cause la probité d'autrui peuvent s'appuyer sur des mobilisations survenant simultanément dans plusieurs espaces sociaux, des mobilisations multisectorielles (Dobry, 1986) liées à la concomitance d'oscillations des perceptions du possible (Rayner, 2005), deux processus qui co-varient pour faire fluctuer la jouabilité de la dénonciation. C'est dans cette perspective que nous entendons rendre compte du vaste mouvement d'indignation qui semble avoir impulsé le scandale des fiches. C'est l'un des principaux apports des travaux consacrés au rôle des émotions dans l'action collective (Goodwin et Jasper, 2004 ; Reddy, 1997 ; Turner et Stets, 2005) que de souligner combien leur expression publique n'a rien d'évident, redevable qu'elle est de configurations, dispositifs, rôles, répertoires et usages différenciés (Sommier, 2010; Mathieu, 2010; Traïni, 2009a et b). Dans notre optique, contrairement aux travaux rapportant les « chocs moraux » à la virulence quasi physique d'une exaspération collective (Jasper, 1997), l'indignation, aussi profonde soitelle, ne suffit pas à elle seule à déclencher une mobilisation ni même à déboucher sur une dénonciation. Encore faut-il que des personnes, qu'elles soient ou non indignées, s'estiment autorisées à la manifester publiquement et trouvent des relais dans plusieurs espaces sociaux. Les formes de son expression publique ne sont pas séparables des relations sociales et, partant, peuvent évoluer (de la répulsion à la simulation, de l'inhibition à l'emphase, etc.) dans le cours de l'action. Est-ce dire que notre approche ne concède aucun rôle aux valeurs dans les scandales? Certainement pas. En tentant de comprendre les logiques sociales faisant que leur transgression puisse être dénoncée ou au contraire soit passée sous silence, nous espérons apporter un peu d'intelligibilité à propos de processus favorisant ou au contraire inhibant leur effectivité, une question que notre enquête pose et sur laquelle nous revenons à l'issue de notre analyse.

9 À partir d'une recherche en cours, nous allons appliquer cette approche relationnelle et processuelle de l'indignation au «scandale des fiches » (1989-1990), le plus important que la Suisse a connu au XXe siècle. Après la « découverte » de 900000 fiches établies par la police fédérale, répertoriant organisations et individus censés constituer une menace pour la sécurité intérieure de l'État, 350000 citoyens demandent à consulter leur fiche et beaucoup fustigent «l'État fouineur ». Nous verrons que l'ampleur inédite de ce scandale ne peut être déduite de celle de l'indignation, d'abord en nuançant la nouveauté des «révélations » concernant la surveillance massive d'une partie de la population, ensuite en montrant comment celle-ci en est soudain venue à être jugée transgressive par une lutte intense de requalification des pratiques de la police politique, et enfin en analysant les conditions sociales de jouabilité de l'indignation, dans un contexte exceptionnellement (é)mouvant.

\section{Savoir ou ne pas savoir : telle n'est pas la question}

Bien que le renseignement soit un domaine d'action publique bénéficiant de protections institutionnelles du secret et caractérisé par une volonté de ne pas (trop) savoir de la part des autres acteurs de l'État (Dobry, 1997: 54), le partage d'informations impliquant potentiellement celui des responsabilités à l'occasion de litiges pouvant mettre en cause l'appareil policier, le système suisse de surveillance de masse était loin de constituer un secret absolu. L'ignorance de l'action policière a pourtant été abondamment avancée durant le scandale. Le ministre de la Justice et de la Police, Arnold Koller, témoignait par exemple de son « effarement » sur les ondes de la Radio suisse romande (journal du soir du 
16 février 1990). Comme beaucoup d'autres hauts responsables, défenseurs ou dénonciateurs, il évoquait une « surprise » qui aurait supposé la méconnaissance totale de telles pratiques. Or quelques brèves recherches historiques remettent facilement en cause l'ignorance affichée du personnel politique et, partant, la thèse d'une chaîne causale révélation/indignation/dénonciation, au profit d'une lecture qui privilégie les dimensions cognitives et contextuelles des dynamiques de (dé)légitimation au cœur du scandale (Dobry, 2002). En dénaturalisant l'occurrence du scandale, on s'autorise alors à mieux concevoir l'énigme que constitue son émergence.

11 La surveillance policière des individus subversifs (comprendre de gauche) était partiellement connue et considérée comme légitime dans le contexte de guerre froide. D'abord, le cercle des insiders n'était de loin pas restreint à une poignée d'agents secrets bernois. En effet, l'organisation décentralisée de la police politique suisse supposait la participation d'un grand nombre de fonctionnaires de police cantonaux chargés du travail de terrain, mais aussi de complicités auprès d'administrations telles celles des postes et téléphones, des compagnies aériennes ou encore des universités qui signalaient leurs étudiants agitateurs. Le Conseil fédéral (gouvernement) lui-même recevait à intervalles réguliers des comptes rendus exhaustifs des observations policières. Les cibles de la police n'étaient pas non plus naïves de la surveillance dont elles pouvaient faire l'objet. Lorsque des membres de la Ligue marxiste révolutionnaire (LMR) découvrirent en 1973 une installation clandestine d'écoute à leur congrès, leur surprise était loin d'être totale : « La découverte [...] d'un micro émetteur ne fit que confirmer certains soupçons et ajouter une preuve de plus à celles que nous avons réunies sur les méthodes qu'utilise quotidiennement la police pour surveiller les militants de la LMR » (La Brèche, 17 mai 1973). Pour sa part, le Manifeste démocratique, association de défense des libertés publiques, dénonçait en 1978 « l'espionnage croissant de soldats, militants anti-nucléaires et autres groupes oppositionnels ", la « multiplication des interdictions professionnelles » et le «fichage systématique » des militants (congrès du Manifeste démocratique des 18 et 19 novembre 1978).

12 Durant les années 1970, la dénonciation de la surveillance policière, d'abord limitée aux milieux communistes, s'élargit à d'autres acteurs (élus socialistes, journalistes, mouvements sociaux, juristes "progressistes ») qui contribuent à une politisation croissante de la question du renseignement intérieur. Entre autres affaires, celle de la LMR suscita en 1973 un vaste débat sur la légalité et la légitimité des écoutes clandestines. Malgré une dénonciation quasi unanime de la presse et de la gauche qui y voyaient une pratique illégale et un abus policier, la majorité de droite du Parlement et le gouvernement estimèrent que le dispositif légal était suffisant et s'en tinrent au statu quo. Le Tribunal fédéral confirma lui aussi le bien-fondé de l'opération policière :

La police chargée de veiller à la sécurité intérieure et extérieure de l'État ne peut se contenter des déclarations publiques d'un groupement qui ne cache pas ses intentions révolutionnaires; elle doit pour le moins chercher à connaître quelles sont les véritables intentions qui peuvent se dissimuler derrière de telles déclarations (ATF $100 \mathrm{Ib} 13$ ).

13 En 1976, c'est l'affaire Cincera qui défraya la chronique. Ce haut gradé de l'armée, membre du Parti radical, avait réuni des milliers de fiches sur des individus réputés subversifs en s'appuyant sur un réseau d'informateurs au sein d'entreprises, de l'armée et de l'administration publique, mais aussi sur ses propres agents infiltrés. La mise au jour de ce bureau privé de renseignement éclaira l'ordinaire de la pratique de fichage et les collusions qui lui assuraient sa légitimité. Au Parlement, la démarche fût soutenue par de 
nombreux députés de droite, dont Rudolf Friedrich, futur conseiller fédéral, qui estimait légitime et même nécessaire que des citoyens ordinaires participent à la lutte antisubversion.

Dans les années 1980, la question du renseignement intérieur revint régulièrement à l'ordre du jour, notamment à Zurich où il fut rendu public que la police politique de la ville avait infiltré une dizaine d'agents dans les mouvements de jeunes. De manière générale, les pratiques policières furent de plus en plus connues. Le Blick, par exemple, tabloïd suisse alémanique, révéla le 23 mars 1989 que la police fichait systématiquement les voyageurs qui se rendaient à l'Est ou qui en venaient et s'offusquait du fait que « notre pays [était] devenu un État policier et de surveillance ». L'Hebdo, premier magazine d'information suisse romand, annonçait le 19 janvier 1989 que le premier policier du pays disposait " de fiches secrètes sur plus d'un million de citoyens suisses », une affirmation qui, pas plus que celles du Blick, n'entraîna de réactions politico-médiatiques. Le Parlement lui-même, par l'intermédiaire de sa commission de surveillance, avait pris connaissance en 1988 de l'existence d'un grand nombre de fiches. Pour autant, ladite commission, présidée par le socialiste Moritz Leuenberger, ne jugea «pas politiquement opportun » de poursuivre son enquête, la majorité de droite accordant sa confiance à la police politique (Kreis, 1993 : 624). Il apparaît dès lors que les révélations concernant la surveillance policière, quand bien même suivies par des dénonciations indignées, ne débouchèrent pas pour autant sur des mobilisations de grande ampleur, d'autant plus que cette pratique était jugée normale par les acteurs clés de l'État durant des décennies, au moins jusqu'en 1990.

Le scandale des fiches émerge d'une affaire qui lui paraît tout à fait étrangère. Fin octobre 1988, Elisabeth Kopp, membre du Parti radical (droite libérale) et cheffe du Département fédéral de justice et police (DFJP) est soupçonnée d'avoir transmis des informations confidentielles à son mari, avocat d'affaires membre du conseil d'administration d'une société financière faisant l'objet d'une enquête pour blanchiment d'argent provenant du trafic de stupéfiants. Devant la pression politique et médiatique, le 10 décembre, elle avoue avoir parlé de l'enquête à son époux. Dénoncé dans une campagne médiatique et politique d'une envergure exceptionnelle, ce comportement est jugé révélateur des collusions entre les autorités politiques, les organes de répression du trafic international de stupéfiants ou de la criminalité organisée et les milieux bancaires ou financiers helvétiques. Lâchée par son parti et le Conseil fédéral, elle annonce sa démission deux jours plus tard, le 12 décembre.

16 La démission d'un ministre en exercice est un fait suffisamment rare et grave pour que la majorité dite «bourgeoise » se convainque de la nécessité d'y apporter une réponse forte. Sur la pression des socialistes, les quatre partis de gouvernement mettent en place en janvier 1989 une commission d'enquête parlementaire (CEP) avec le mandat très large d'enquêter sur l'ensemble du Département de la ministre démissionnaire. Des indices laissent penser que le Parti socialiste y a aussi vu l'occasion de faire la lumière sur les activités de la police politique (Liehr, 2014: 248), qui en dépendent. Riposte institutionnelle censée confiner la controverse à une arène restreinte durant plusieurs mois, l'instauration d'une CEP, dont l'usage est rarissime en Suisse (un seul précédent en 1964), participe aussi d'une dramatisation des enjeux transformant les perceptions relatives à la jouabilité de la dénonciation des fichiers. 


\section{Transgression ou pas? Le recadrage de la surveillance politique}

17 La CEP publie son rapport le 24 novembre 1989 au terme de plusieurs mois de travail. Très attendu et largement médiatisé, ce volumineux document porte pour l'essentiel sur les éléments à l'origine de l'affaire Kopp. Un seul chapitre est consacré aux activités de la police politique, dans lequel il est indiqué que celle-ci détient un fichier comprenant quelque 900000 fiches, les deux tiers concernant des étrangers, le reste se divisant pour moitié entre des personnes d'une part, des organisations ou des événements d'autre part. Il est également mentionné que cette surveillance portait principalement sur des groupements politiques de gauche et écologiques, pacifistes, féministes, antimilitaristes et antinucléaires, ainsi que sur des parlementaires, des journalistes ou des membres de comité d'initiatives, alors que l'extrémisme de droite n'y figure que depuis deux ans. Pour autant, si le rapport émet un regard critique sur le renseignement politique tel que pratiqué par la police fédérale (absence de pertinence, de systématique et de contrôle politique, dérives, lacunes, carences, appréciation «étroite» ou "simpliste» de la menace, et manque de professionnalisme, etc.), il n'en considère pas moins son existence comme légitime et nécessaire, contribution «en principe positive » à la protection de l'État. Dans l'ensemble, la tonalité du rapport est loin de présenter une image scandaleuse $\mathrm{du}$ renseignement politique intérieur, mais il en officialise certaines pratiques. Les conclusions ne portent du reste pas sur cette question, qui n'est évoquée qu'incidemment, par une sorte d'obiter dictum appelant à rétablir la légitimité de l'État.

18 La réception de ce rapport par les médias est, dans un premier temps, mitigée et très majoritairement focalisée sur l'affaire Kopp, ainsi que sur des propositions de réformes soumises à l'Assemblée fédérale pour sa prochaine session, une dizaine de jours plus tard. Cette période est alors marquée par une lutte intense de recadrage de la situation autour des activités de la police politique. Les partis de la droite traditionnelle, radicale, libérale, démocrate-chrétienne et agrarienne très largement majoritaires au Parlement - ces partis dits «bourgeois » occupent près de $70 \%$ des 200 sièges au Conseil national et 40 sièges sur 46 au Conseil des États - saluent le «travail remarquable » de la Commission qui, hors certaines faiblesses, erreurs ou négligences, ne révèlerait rien qui puisse être constitutif d'une crise de régime, une évaluation qui est largement relayée dans presque toute la presse helvétique. On lit par exemple dans Le Matin du 25 novembre 1989 que le rapport, pourtant «sans complaisance, ne révèle pas de scandale». La Neue Zürcher Zeitung (NZZ) et le Journal de Genève, deux quotidiens reconnus comme porte-parole de la droite «bourgeoise", radicale libérale, n'évoqueront la question des fichiers qu'une semaine plus tard.

19 Journal zurichois concurrent de la NZZ, le Tages Anzeiger fait cependant exception. Le 25 déjà, il publie un article intitulé La police politique ne fichait que la gauche, entièrement focalisé sur le chapitre du rapport consacré à la police politique, dans lequel apparaît l'expression schnüffeln (renifler), emblème des mobilisations à venir. Comparant la police fédérale à la Stasi, l'éditorial opère un déplacement : « La CEP apporte certes un éclairage approfondi sur l'affaire Kopp, mais elle tombe sur un nouveau scandale». Cette couverture discordante est amplifiée le même jour par le Blick, qui évoque en première page, selon un registre plus émotionnel propre à la presse dite " à sensation », le « choc » des révélations de la CEP, ainsi que le caractère «effrayant» des 900000 fiches 
découvertes, tout en ironisant sur l'attitude "conciliante» du Parti radical : «Qu'a-t-il appris, s'il vous plait, du scandale?» Trois jours plus tard, ce même journal consacre sa première page au fichage des parlementaires, évoquant différentes réactions indignées, y compris celle d'un député démocrate-chrétien qualifiant cette pratique de " cochonnerie de haut vol » (ausgekochte Schweinerei), une prise de position peu attendue d'un colonel de l'armée appartenant au même parti qu'Arnold Koller, conseiller fédéral responsable du dossier, mais qui indique aussi certaines défections au sein du camp "bourgeois ». À partir du 29, le même Blick exige la révocation des chefs de la police fédérale renommée pour l'occasion "Schnüffel-Abteilung" (section de reniflage). Il signale l'« indignation généralisée » provoquée par les récentes « révélations » et invite ses lecteurs à solliciter l'accès à leur fiche en publiant une lettre type adressée à Arnold Koller lui-même.

Avec retard, les autres journaux changent de focale, principalement sous l'effet de la mobilisation des parlementaires socialistes et écologistes, ainsi que de dirigeants syndicaux qui, dès le 27 novembre, commencent à dénoncer dans la presse l'«État fouineur » (Schnüffelstaat) tout en réclamant l'accès à leur propre fiche. Dans un processus d'alignement des différents titres de presse, l'affaire Kopp est peu à peu reléguée au second plan, alors que se multiplient les articles à propos des fiches, parfois très critiques comme celui du Bund du 29 novembre (journal bernois pourtant assez conservateur) intitulé Glasnost anstatt Schnüffelstaat. Sous pression, Arnold Koller est contraint à des concessions. Le 30 novembre, "comprenant l'indignation des citoyens", il admet le principe de leur accès limité aux fichiers, sous certaines conditions. Cette riposte face aux critiques qui s'intensifient est reprise par tous les titres au niveau national. Elle vise surtout à relativiser la signification de la surveillance politique, le conseiller fédéral riant devant la presse en exhibant une fiche établie à son nom comme preuve du "dilettantisme » des fonctionnaires de police, un cadrage qui éprouve des difficultés à s'imposer, du moins dans un premier temps, mais qui sera souvent mobilisé dans le cours du scandale pour affaiblir les dénonciateurs.

21 En Suisse romande (francophone), il revient surtout à L'Hebdo d'amplifier la controverse. Son édition du 30 novembre consacre plus d'une dizaine de pages au rapport, où sont opposés le laxisme des autorités dans le domaine du blanchiment d'argent sale et le zèle de la police politique à l'égard des organisations et individus « progressistes ». L'éditorial met en cause «des journaux prestigieux, qui osent minimiser l'affaire, après avoir systématiquement attaqué leurs confrères qui, non sans risques, ont enquêté depuis des mois sur ce terrain brûlant». Le ton de l'hebdomadaire ne varie guère au fil des semaines : «choc ", " scandale ", « indignation ", « colère » et " protestation », autant de termes qui égrènent le fil d'enquêtes tout entières animées par une perspective de dénonciation nettement moins présente dans la plupart des autres titres de la presse nationale.

Dans l'ensemble, les médias helvétiques hésitent encore dans leur cadrage comme en atteste une revue de la presse suisse alémanique publiée le 2 décembre dans le Journal de Genève. Sous le titre Tempête dans un verre d'eau, repris de l'un des titres recensés, le quotidien genevois évoque "bon nombre de commentateurs reprochant à certains parlementaires fédéraux d'exploiter politiquement cette affaire ». Ainsi, selon l'auteur de cette revue de presse, la Solothurner Zeitung

se moque des parlementaires fédéraux [...], estimant tout de même curieux que ces politiciens choqués aient mis si longtemps pour se rendre compte de leur état de choc [alors que] personne n'a bronché il y a une année lorsque la commission de gestion du Conseil national avait critiqué le fichier de la police fédérale. 
On le voit, la controverse porte sur la réalité de l'indignation suscitée par les «révélations» du rapport: les uns s'indignent de l'indignation des autres, accusés d'instrumentaliser un "problème » connu de longue date, alors que ceux-ci s'indignent que les premiers ne le soient pas à l'épreuve de faits désormais dévoilés, objectivés dans le rapport et reconnus par tous. C'est dire que l'indignation associée aux fichiers ne va pas de soi dans les premiers moments du scandale. Que celle-ci soit feinte ou réelle, telle n'est pas ici la question d'un point de vue sociologique, car l'enjeu porte alors sur la légitimité, et donc la jouabilité, de son expression publique en regard des transgressions supposées, une question qui divise aussi bien les politiques que les journalistes, deux catégories d'acteurs inscrits dans des univers sociaux très concurrentiels.

C'est en effet à la faveur d'une conjonction contingente de plusieurs événements que se transforment les perceptions de la situation politique et que se comprend la mobilisation d'acteurs minoritaires s'indignant ouvertement du fichage politique, une pratique certes connue, parfois dénoncée, mais finalement peu controversée en raison des soutiens haut placés dont elle a bénéficié. Si la chute du mur de Berlin le 9 novembre 1989 réduit l'impact de la symbolique politique attachée à la guerre froide, sur le plan intérieur, le scrutin du 26 novembre (soit deux jours après la publication du rapport de la CEP) sur l'initiative populaire demandant l'abolition de l'armée stupéfie littéralement la classe politique, tant le résultat du vote ( $35,6 \%$ de suffrages favorables) est perçu comme une atteinte à la légitimité d'une institution quasi sacralisée. Il ne fait par ailleurs aucun doute que l'affaire Kopp a fragilisé la crédibilité des partis "bourgeois», soupçonnés d'entretenir des collusions avec les milieux bancaires. Tous ces éléments pèsent sur la session parlementaire qui s'ouvre le 27 novembre par l'examen d'un projet de loi sur le blanchiment d'argent sale, les parlementaires de gauche étant nettement mis en minorité sur leur proposition de réprimer aussi la négligence des institutions bancaires (et non uniquement le délit intentionnel). Il en va de même à propos du budget de l'armée que refuse le groupe socialiste ou encore d'un projet de loi sur l'objection de conscience. Le $1^{\text {er }}$ décembre, pratique inédite au Parlement helvétique, une cinquantaine de députés socialistes adressent tout à tour la même question à Arnold Koller, lui demandant si le Ministère public fédéral avait établi une fiche les concernant et si le Conseil fédéral acceptait de la remettre pour consultation.

C'est donc dans un contexte en voie de polarisation que s'ouvre le débat parlementaire sur le rapport de la CEP. Il se déroule sur plusieurs séances, les 6, 7, 11 et 13 décembre, preuve d'une mobilisation exceptionnelle sur des « révélations » que le Conseil fédéral (1990 I 848-867) et les partis « bourgeois » minimisent, comme le rapporteur (libéral) de la Commission, estimant que celle-ci «n'a pas mis au jour un énorme scandale». Et il ajoute : «Certains sont peut-être déçus de l'absence de scandale. Mais comment peut-on souhaiter que l'État ou ses institutions soient éclaboussés par un scandale?» (Assemblée fédérale, 1989 : 1992) À droite, les parlementaires demandent que « soit gardé le sens des proportions » et « reconnue l'absence d'une crise de régime » ou encore que « les faits ne soient pas considérés émotionnellement, mais avec objectivité ». Dans la tonalité du rapport de la CEP, ces prises de position admettent certaines erreurs, parfois graves, mais surtout imputables à des personnes, et soulignent dans le même temps que les réformes proposées seront de nature à rétablir la confiance des citoyens envers leurs institutions. Tout en souhaitant que le débat ne se focalise pas sur les fiches, ces députés sont unanimes à revendiquer la nécessité de la police politique, notamment dans la lutte contre le terrorisme, moyennant un encadrement juridique plus précis. À gauche, les 
critiques sont virulentes: "Que vous faut-il pour parler de scandale?», demande un député socialiste, suivi par plusieurs dizaines d'interventions dénonçant les collusions entre milieux bancaires et politiques, parlant de "prostitution fiscale», évoquant la «perversion » de la protection de l'État, qualifiée de « cimetière de la guerre froide qui ne protège ni de la drogue ni de l'extrême droite ». Ces parlementaires sont tout aussi unanimes pour exiger un changement de «culture politique» impliquant alors la suppression de la police politique. Le président du parti socialiste déclare dans la presse ( Tages Anzeiger du 9 décembre) que ces débats ne sauraient se réduire à un simple « rituel d'indignation" (Empörungsritual), mais qu'il faut en tirer la leçon: "Au regard des changements en Europe et en Suisse, cette Stasi-Politik ne peut plus durer ». En fin de compte, les propositions de réforme censées mettre un terme à la crise sont adoptées. Les jours qui suivent, la presse nationale (y compris le Blick) n'évoque plus guère l'affaire Kopp, qui parait révolue, ni même les controverses à propos des fiches. Au sortir des débats, le 13 décembre, les mobilisations se limitent encore à l'arène parlementaire et l'expression publique de l'indignation semble refluer sur le microcosme politique et quelques rédactions de presse plus critiques. Comment dès lors comprendre l'élargissement du litige?

\section{Le scandale comme variation émergente de la jouabilité de la dénonciation}

26 À partir de janvier 1990, pour un nombre croissant d'acteurs et dans plusieurs univers sociaux, l'indignation publique contre «l'État fouineur» devient beaucoup plus exprimable et partageable que d'ordinaire, au point de les porter à des prises de position longtemps réfrénées. Cette "partageabilité » constitue d'ailleurs l'une des principales facettes de «l'expérience émotionnelle» (Rimé, 2005 : 87). Interrogé par un journaliste sur sa découverte des fiches, Moritz Leuenberger n'hésite plus à emprunter un registre émotionnel en disant avoir été «horrifié » (L'Hebdo, 11 janvier 1990), une désinhibition tributaire de son évaluation du rapport de force politique : «La pression publique était telle qu'il était difficile de faire autrement» (que de les dénoncer), alors même qu'il considérait la dénonciation comme impossible quelques mois plus tôt. Pour comprendre ce revirement, il faut l'inscrire dans la dynamique qui prend place entre décembre 1989 et mars 1990, faite de mobilisations multisectorielles et de nouvelles estimations de la situation autour de la dénonciation publique du fichage. Cette concomitance de fortes oscillations des perceptions du possible est d'ailleurs ce qui fait du scandale des fiches un événement (Rayner, 2005 ; Romano, 1998).

Ce processus génère sa propre dynamique, crée de nouvelles opportunités et contraintes, soit autant d'incitations à agir autrement. Nous tenons là la propriété émergente du scandale dont les ressorts apparaissent et changent au cours de l'action. Des revendications longtemps jugées déraisonnables (demander d'accéder à sa fiche, l'abolition de police politique, le boycott du $700^{\mathrm{e}}$ anniversaire de la Confédération ou du recensement), car vouées à l'échec, se révèlent soudain possibles dans une dynamique où les «effets» rétroagissent sur les "causes». Ainsi, la revendication d'un groupe de parlementaires (cause) débouche sur l'autorisation à consulter sa fiche (effet), une concession d'un conseiller fédéral qui va très vite inciter ces derniers à revendiquer l'abolition de la police politique (rétroaction). Le sens que les acteurs donnent à leur environnement évolue très vite parce que des rétroactions en série engendrent de la 
disproportion : circonscrites début décembre à quelques élus et journalistes, les demandes de consultation des fiches proviennent très vite de centaines d'activistes puis, jour après jour, de dizaines de milliers de citoyens, jusqu'à atteindre le 31 mars 1990, délai fixé par les autorités, le chiffre stupéfiant de 350000 demandes, là où les dirigeants du Ministère public en prévoyaient 3 ou 4000 . Cette augmentation vertigineuse et imprévue du nombre de ces demandes constitue l'un des effets de seuil attestant aux yeux des différents acteurs de l'intensification du scandale. Parmi les autres indices d'un basculement susceptible de galvaniser les uns (les dénonciateurs) et d'inquiéter les autres (les représentants des autorités), figurent l'emballement de la " couverture médiatique ", des tirages pour le moins inhabituels (les 300000 exemplaires de Fichen Fritz/Fiche \& Fouine, journal du comité contre l'État fouineur fondé le 19 janvier 1990, les 30000 exemplaires vendus dès janvier du rapport de la CEP, après un premier tirage à 4000 exemplaires, et les 25000 exemplaires du livre Schnüffelstaat Schweiz), la participation massive à la manifestation nationale de Berne du 3 mars 1990 (30 000 personnes alors que les organisateurs en attendaient 10000 et qui fut suivie d'émeutes), des résultats d'enquêtes d'opinion, tel ce sondage selon lequel $« 72 \%$ des Suisses souhaitent une enquête sur le Département militaire fédéral» (SonntagsBlick, 18 février 1990) ou celui donnant lieu au titre Halte à la fichomanie! Pour une majorité des personnes, il faut les limiter, voire les supprimer (Le Matin, 14 mars 1990).

Dans plusieurs espaces sociaux, une refonte du concevable est à l'œuvre. Parlementaires, militants, syndicalistes, journalistes, universitaires, artistes et beaucoup de «citoyens ordinaires » révisent leurs estimations quant à l'opportunité de s'offusquer publiquement de la politique de surveillance de la population instituée depuis des décennies: conjointement, souvent par coordination tacite, ils s'autorisent ce qu'ils s'interdisaient jusque-là et se mobilisent pour dénoncer « l'État fouineur ». Cette dynamique du scandale s'apparente à un jeu de miroirs où des acteurs s'observent, se testent et s'influencent continûment (Rayner, 2007). Cette dimension spéculaire est une propriété essentielle du scandale (Rayner, 2015). L'autosuggestion collective où les uns et les autres se convainquent de la jouabilité de la dénonciation produit une rupture de sens. Miser sur la "scandalisation» n'a plus rien d'incongru. La dénonciation de "l'État fouineur " s'imposant comme définition dominante de la situation, il s'avère même coûteux de ne pas s'offusquer (Rayner, Thétaz et Voutat, 2016).

Dans la mesure où les pratiques d'espionnage visaient aussi nombre de journalistes, la reprise massive par de nombreux titres de presse, tous profils confondus, du cadrage promu par le comité contre l'État fouineur («scandale des fiches ", "scandaleux », «État fouineur ", " renifleur», "fichomanie », " crise de confiance »), est lourde de menaces pour les élus "bourgeois", envers lesquels des journalistes se font beaucoup plus critiques qu'à l'accoutumée. L'Hebdo du 22 mars 1990 titre sa une avec Scandale des fichiers : l'État-flic et l'éditorial raille "l'indignation vertueuse » des ministres. L'emprise de cette définition dominante de la situation peut se mesurer à la manière dont elle infléchit les prises de position publiques : d'un côté, les opposants au fichage semblent exaltés jusqu'à réclamer ouvertement ce qui relevait auparavant de l'impossible (l'abolition de la police politique), de l'autre, des porte-parole des partis dits "bourgeois » se sentent mis en demeure de se repositionner, notamment en s'appropriant à leur façon cette définition tendant à stigmatiser ce qui, jusque-là, allait de soi. Cette force contraignante du scandale pousse les gouvernants à des concessions. À plusieurs reprises, le conseiller fédéral Arnold Koller reconnaît que "l'indignation des citoyens est justifiée ». Ainsi dans le 
Journal de Genève du 6 mars 1990: "Des milliers de citoyennes et citoyens sont, à juste titre, indignés d'avoir été surveillés, soupçonnés et enregistrés dans l'exercice de leurs droits démocratiques. Le Conseil fédéral comprend et partage cette consternation ». Désormais, cette qualification négative des pratiques de la police politique s'impose: «Déjà, les quatre partis gouvernementaux se sont indignés après la découverte des nouvelles fiches » (journal du soir de la Radio suisse romande du 16 février 1990) ; «Ce ne sont pas des excuses. Mais le Conseil fédéral regrette les fautes commises dans le domaine de la protection de l'État et de la sécurité militaire. Il comprend l'indignation des citoyens » (Le Matin, 22 février 1990). À propos des membres de la CEP, même le Journal de Genève, le 9 mars 1990, concède : «Face à l'opinion publique, reconnaissons qu'il leur est difficile de procéder autrement, et que nager à contrecourant en pleine crise de confiance pourrait être mal interprété ». Tous ces éléments témoignent de la force sociale d'une configuration conduisant à un alignement collectif (Ermakoff, 2008), du moins en apparence, sur un cadrage qui devient temporairement dominant.

La multiplication des "révélations» de certaines des pratiques de surveillance vient accréditer le cadre interprétatif dominant en termes d' «État dans l'État », de surveillance policière et de contrôle social antidémocratiques. C'est surtout l'extension des actions collectives qui permet cette conductivité : piquet de protestation « permanent » devant le siège du Ministère public, manifestations devant les sièges des polices cantonales, etc. Durant le premier trimestre 1990, les rapports de force et de sens semblent s'être inversés, la honte et l'indignation changent de camp : des « contestataires » parviennent à mobiliser en masse et à imposer leur définition de la situation, des "subversifs" longtemps stigmatisés peuvent soudain fustiger à bon droit leurs "oppresseurs», des conseillers fédéraux et des dirigeants de la police semblent décontenancés par ce phénomène d'auto-amplification dont nul ne connaît l'issue ; indétermination qui a d'ailleurs passablement tourmenté ses principaux protagonistes, pris dans une tournure inédite de leurs échanges. C'est aussi pourquoi cette conjoncture, typique des crises politiques, s'avère plus favorable à l'expression publique des émotions (effarement, indignation, colère, peur, joie, honte, etc.), autant d'efforts d'adaptation à une situation exceptionnellement (é)mouvante. Devenue l'une des porte-parole du Comité contre l'État fouineur en Suisse romande, la députée socialiste Françoise Pitteloud fait de l'indignation le leitmotiv de son intervention devant la foule venue manifester le 3 mars 1990 contre le fichage sur la place fédérale à Berne :

C'est en votre nom que j'exprime aujourd'hui l'indignation qui a saisi le pays tout entier devant le scandale que représente le fichage de milliers de personnes par la police politique. Au Conseil fédéral dans son ensemble, nous disons ceci : plutôt que de vous indigner que l'on compare ces pratiques sinistres à celles de la Stasi, prenez des mesures afin qu'elles ne puissent se reproduire! La police politique doit être supprimée, elle est indigne d'une démocratie. Cessez d'avoir peur de nous! Nous sommes le peuple ! (Komitee Schluss mit dem Schnüffestaat, 1990 : 15-16)

31 De l'instrumentalisation la plus cynique à l'expression la plus franche, le rapport à l'indignation peut évoluer dans le cours de l'action : il change d'un individu à l'autre et, chez un même individu, d'une séquence à l'autre. Ainsi, des activistes parmi les moins surpris par la « révélation » des fichiers ont pu (sur)jouer l'indignation afin de mobiliser des soutiens tout en étant traversés par une indignation «sincère " au moment où ils manifestent ensemble dans les rues de Berne. L'effervescence qui peut surgir de l'action collective n'est pas sans effets sur ses participants et ses observateurs. « La manifestation s'est poursuivie vers $15 \mathrm{~h}$ sur la place Fédérale. Les banderoles et les discours clamaient 
l'indignation. » (Le Matin, 4 mars 1990) Cependant, en dépit de cette soudaine légitimation de la stigmatisation du fichage, les mobilisations vont très rapidement refluer à partir d'avril 1990 : d'un côté, les « indignés » se focalisent sur la récolte des 100000 signatures nécessaires à leur initiative populaire « contre l'État fouineur » et les journalistes cessent d'en faire une priorité ; de l'autre, les dirigeants du camp «bourgeois » parviennent à recloisonner le litige au sein du Parlement, notamment pas l'intermédiaire d'une nouvelle CEP, cette fois sur le Département militaire, et d'un projet de loi sur le renseignement intérieur, ripostes institutionnelles sous forme de concessions qui concourent à la canalisation du conflit. La retombée et la sectorisation des mobilisations rendent à nouveau l'expression publique de l'indignation moins jouable, voire incongrue. En 1998, dans un contexte nettement moins conducteur, l'initiative populaire sera rejetée par $75 \%$ des votants.

\section{Conclusion}

Nous avons montré combien le scandale ne peut se réduire à la virulence d'une indignation qui lui serait préalable. Celle-ci se déploie si - et seulement si - des acteurs se sentent autorisés, par ce qu'ils perçoivent du contexte, à agir publiquement en son nom, peu importe de ce point de vue qu'elle soit feinte ou véritable. Bien avant le scandale, des parlementaires de gauche connaissent l'étendue du système de surveillance politique qui les visent mais, jugeant le rapport de force politique défavorable, estiment «peu opportun" de s'en émouvoir. C'est à la faveur de ce qu'ils perçoivent comme une transformation du contexte (chute du mur de Berlin, résultat surprenant d'une initiative populaire, articles de presse dénonciateurs, etc.) qu'ils se permettent soudain ce qu'ils s'interdisaient jusque-là : crier au scandale. Leur enrôlement dans les mobilisations constitutives du scandale les porte à adopter le registre de la dénonciation indignée, ce qui n'est pas sans effet sur leur propension à éprouver "sincèrement » de l'indignation, en particulier durant les phases les plus intenses de l'action collective.

Loin de suivre un implacable enchaînement séquentiel (révélation, indignation, dénonciation, scandalisation), le scandale constitue un processus ouvert et non linéaire caractérisé par une causalité spirale : à la fois cause et effet, l'indignation collective émerge d'une recombinaison des relations entre les différents protagonistes. Reliés par des jeux spéculaires, dénonciateurs et dénoncés, ils s'appuient les uns sur les autres pour ressentir jusqu'où aller dans l'expression de leurs émotions et prises de position. Voilà pourquoi les émotions évoluent dans le cours de l'action, au gré de rapports de force, ce qui ne signifie pas pour autant qu'il faille adopter une perspective instrumentale. Prendre pour objet les émotions, ici l'indignation, la colère ou la honte, expose à deux écueils : d'une part, la perspective stratégiste de l'acteur rationnel, où les émotions sont maîtrisées par le calcul et guidées par l'intérêt (un biais intentionnaliste à l'œuvre dans la question "à qui profite le scandale?»); d'autre part, la perspective sémiotique (y compris celle qualifiée de corporelle) consistant à prendre celles-ci au pied de la lettre, comme l'extériorisation sans aspérités d'états intérieurs redevables d'une adhésion sans faille à des valeurs et autres croyances. Dans un cas comme dans l'autre, la démarche tend à décontextualiser les émotions, à les couper des relations où elles surgissent et se transforment tout en les affectant.

Solidaire du contexte, et, partant, de la façon dont les acteurs se représentent le faisable, l'indignation n'est donc pas séparable de l'action. Sincère ou simulée, elle peut revêtir 
toute une gamme de rapports et de modulations. Cette conception du scandale vise à rendre compte de l'interdépendance, cognitive et émotionnelle, d'acteurs sensibles aux fluctuations mutuelles de leurs perceptions du possible. Ce déplacement de la focale sociologique des transgressions vers les dynamiques de mobilisation autour de leur réprobation permet de saisir ce que la confusion très commune du scandale et des faits présumés scandaleux occulte, à savoir les variations, parfois très spectaculaires, de la jouabilité de la dénonciation. En outre, cette exploration processuelle et relationnelle des ressorts complexes de la scandalisation donne à comprendre pourquoi le scandale constitue l'exception plutôt que la règle : il ne prend place que si des acteurs parviennent à qualifier des pratiques de déviantes et à mobiliser simultanément, entre et dans plusieurs espaces sociaux, des soutiens massifs à leur campagne de moralisation. Si les scandales sont aussi des moments de rappel à des valeurs censées être communément admises par la collectivité, pour le sociologue Bourdieu (1994), il importe peu que l'indignation morale soit sincère ou feinte, fidèle ou intéressée, vertueuse ou cynique. Ce qui compte, c'est son expression publique. C'est dire qu'une démarche relevant de l'éthique publique, à partir du moment où elle s'interroge sur ce qui rend possible les transgressions, peut aussi s'intéresser, avec les sciences sociales, à ce qui rend possible leur dénonciation. On peut imaginer ici la mise en place de dispositifs favorisant la transparence au détriment de la culture du secret entourant les pratiques policières en matière de renseignement politique ou contre les collusions qui, dans d'autres domaines (en politique ou en économie), sont à l'origine de la violation des règles normatives. On peut aussi penser à des mécanismes de protection des lanceurs d'alerte, qui peuvent renforcer la jouabilité des dénonciations et, par extension, inciter au respect de certaines valeurs mises en cause dans les scandales. C'est probablement sur ces problématiques que les sciences sociales peuvent apporter une contribution à l'éthique publique.

\section{BIBLIOGRAPHIE}

ALEXANDER, Jeffrey C. (1988), « Culture and political crisis: Watergate and Durkheimian sociology », dans Jeffrey ALEXANDER (dir.), Durkeimian sociology: cultural studies, Cambridge, Cambridge University Press.

ASSEMBLÉE FÉDÉRALE (1989), « Événements survenus au DFJP ». Bulletin officiel, 1989, Volume V, Conseil national, séances des 6, 7 et 11 décembre 1989, 1988-2010, 2011-2029, 2037-2050 ; Conseil des États, séance du 13 décembre 1989, 790-810.

BARTHE, Yannick et al. (2013), « Sociologie pragmatique mode d'emploi », Politix, revue des sciences sociales du politique, $\mathrm{n}^{\circ} 103$, p. 174-204.

BLIC, Damien DE et Cyril LEMIEUx (2005), «Le scandale comme épreuve. Eléments de sociologie pragmatique ", Politix, revue des sciences sociales du politique, $\mathrm{n}^{\circ} 71, \mathrm{p} .9-38$, [en ligne], [https:// www.cairn.info/revue-politix-2005-3.htm], (29 décembre 2016).

BOLTANSKI, Luc (1990), L'Amour et la Justice comme compétences, Paris, Métailié. 
BOLTANSKI, Luc et Laurent THÉVENOT (1991), De la justification. Les économies de la grandeur, Paris, Gallimard.

BOLTANSKI, Luc et al. (dir.) (2007), Affaires, scandales et grandes causes. De Socrate à Pinochet, Paris, Stock.

BOURDIEU, Pierre (1994), « Un fondement paradoxal de la morale », Raisons pratiques. Sur la théorie de l'action, Paris, Seuil, p. -244.

BOURDIEU, Pierre (1997), Méditations pascaliennes, Paris, Seuil.

CHAMPAGNE, Patrick et Dominique MARCHETTI (1994), «L'information sous contrainte. À propos du scandale du sang contaminé », Actes de la recherche en sciences sociales, 101/102, p. 40-62.

COMMISSION D’ENQUÊTE PARLEMENTAIRE (1990), « Événements survenus au DFJP. Rapport du 22 novembre $1989 »$, Feuille fédérale, 1990 I 593-847.

CONSEIL FÉDÉRAL (1990), « Événements survenus au DFJP. Prise de position sur le Rapport de la Commission d'enquête parlementaire du 4 décembre 1989 », Feuille fédérale, 1990 I 848-867.

DAMPIERRE, Éric DE (1954), « Thèmes pour l'étude du scandale », Annales ESC, vol. 9, nº 3, p. 329-336.

DANIEL, Justin (1992), « Les démocraties pluralistes face aux scandales politiques : l'Irangate, les

“affaires" Flick et Carrefour du développement ", Revue française de science politique, vol. $42, \mathrm{n}^{\circ} 6$, p. 981-1007.

DOBRY, Michel (1986), Sociologie des crises politiques, Paris, PFNSP.

DOBRY, Michel (1993), « Logiques de la fluidité politique », dans François CHAZEL (dir.), Action collective et mouvements sociaux, Paris, PUF, p. 177-182.

DOBRY, Michel (1997), « Le renseignement dans les démocraties occidentales. Quelques pistes pour l'identification d'un objet flou ", Cahiers de la sécurité intérieure, vol. 30, p. 53-85.

DOBRY, Michel (2002), « Valeurs, croyances et transactions collusives. Notes pour une réorientation de l'analyse de la légitimation des systèmes démocratiques ", dans Javier SANTINO (dir.), À la recherche de la démocratie, Paris, Karthala, p. 103-120.

DURKHEIM, Émile ([1960] 1912), Les formes élémentaires de la vie religieuse, Paris, PUF.

ERMAKOFF, Ivan (2008), Ruling Oneself Out. A Theory of Collective Abdication, Durham, Duke University Press.

GARRIGOU Alain (1992), « Le boss, la machine et le scandale. La chute de la maison Médecin », Politix, revue des sciences sociales du politique, vol. $5, \mathrm{n}^{\circ} 17$, p. 7-35.

GARRIGOU Alain (1993), « Le scandale comme mobilisation », dans François Chazel (dir.), Action collective et mouvements sociaux, Paris, PUF, p. 183-191.

GOODWIN, Jeff et James JASPER (2004), Rethinking Social Movements: Structure, Meaning and Emotion, Lahame, Rowman \& Littlefield Publishers.

JASPER, James (1997), The Art of Moral Protest. Culture, Biography and Crativity in Social Movements, Chicago, University of Chicago Press.

HAMIDI, Camille (2009), « Le scandale n'aura pas lieu ou l'affaire Pechiney saisie par la presse », Revue française de sociologie, vol. 50, $\mathrm{n}^{\circ} 1, \mathrm{p}$. 91-121.

KOMITTEE SCHLUSS MIT DEM SCHNÜFFESTAAT (dir.) (1990), Schnüffelstaat Schweiz, Berne, s.n. 
KREIS, Georg (dir.) (1993), La protection politique de l'État en Suisse. L'évolution de 1935 à 1990, Berne, Haupt.

LIEHR, Dorothee (2014), Skandal und Nation, Marburg, Tectum Verlag.

LOWI, Theodor (1988), « Foreword », dans Andrei S. MARKOVITS et Mark SILVERSTEIN (dir.), The politics of scandal, New-York, Holmes et Meier, p. 7-12.

MATHIEU, Lilian (2010), « Les ressorts sociaux de l'indignation militante. L'engagement au sein d'un collectif départemental du Réseau éducation sans frontière », Sociologie, I, 3, p. 303-318.

RAYNER, Hervé (2005), Les scandales politiques, l'opération « Mains propres » en Italie, Paris, Michel Houdiard Éditeur.

RAYNER, Hervé (2007), Dynamique du scandale, Paris, Le cavalier bleu.

RAYNER, Hervé (2015), « De quoi les scandales sont-ils faits? », Traverse, 2015/3, p. 33-45.

RAYNER, Hervé et Fabien THÉTAZ (2015), Compte-rendu de « À l'épreuve du scandale », Politix, revue des sciences sociales du politique, $\mathrm{n}^{\circ}$ 71, 2005 ; et de Luc BOLTANSKI et al. (dir.), Affaires, scandales et grandes causes. De Socrate à Pinochet, Paris, 2007, Traverse, 2015/3, p. 180-183.

RAYNER, Hervé, Fabien THÉTAZ et Bernard VOUTAT (2016), « Les institutions politiques suisses à l'épreuve : le scandale des fiches (1989-1990) », dans Malik MAZBouRI et François VALLOTTON (dir.), Scandale \& Histoire, Lausanne, Antipodes, p. 81-98.

REDDY, William (1997), « Against Constructionism: the historical ethnography of emotions ", Current Sociology, vol. 38, n 3, p. 327-351.

RIMÉ, Bernard (2005), Le partage social des émotions, Paris, PUF.

RoMAno, Claude (1998), L'événement et le monde, Paris, PUF.

SHERMAN Lawrence W. (1989), «The mobilization of scandal », dans Arnold HEIDENHEIMER, Michael JOHNSTON, Victor LEVINE (dir.), Political corruption. À Handbook, New Brunswick, Transaction Publishers, p. 887-912.

SOMMIER, Isabelle (2010), « Les états affectifs ou la dimension affectuelle des mouvements sociaux », dans Olivier FILLIEULE, Eric AGRIKKOLIANSKY et Isabelle SOMMIER, Penser les mouvements sociaux, Paris, La découverte, p. 185-202.

THOMSON, John B. (2000), Political Scandal, Cambridge, Polity.

TRAÏNI, Christophe (2009a), « Choc moral », dans Olivier FILLIEULE, Lilian MATHIEU et Cécile PÉCHU, Dictionnaire des mouvements sociaux, Paris, Sciences Po, p. 101-107.

TRAÏNI, Christophe (dir.) (2009b), Émotions... Mobilisations !, Paris, Sciences Po.

TRIBUNAL FÉDÉRAL (1974), Arrêt du 8 mars 1974, Affaire LMR contre DFJP, ATF 100 Ib 13.

TURNER Jonathan et Jan STETS (2005), The Sociology of Emotions, Cambridge, Cambridge University Press.

\section{RÉSUMÉS}

À partir d'une étude de cas portant sur le « scandale des fiches » (1989-1990), qui mit en cause les pratiques de surveillance de la police politique suisse, nous montrons que, contrairement à ce qu'affirment nombre de travaux, l'ampleur du scandale ne peut-être déduite de celle de 
l'indignation pas plus que cette dernière ne peut être rapportée à la gravité supposée de transgressions. Un scandale n'émerge que si des acteurs, à partir de leur évaluation de la situation et de leurs perceptions du possible, s'autorisent à exprimer publiquement une indignation, qu'elle soit feinte ou sincère, et parviennent à imposer une qualification déviante des pratiques concernées entraînant des mobilisations dans plusieurs espaces sociaux. En déplaçant la focale sur la dynamique émergente de mobilisations multisectorielles, nous plaidons pour une approche relationnelle et processuelle de la dimension émotionnelle du scandale, inséparable de sa dimension cognitive.

On the basis of a case study of the "secret files scandal" (1989-1990), which challenged the surveillance practices of the Swiss political police, we show that, contrary to what many scholars claim, the extent of a scandal cannot be deduced from the importance of indignation nor the latter can be inferred from the supposed gravity of transgressions. A scandal emerges only if actors, relying on their assessment of the situation and their perceptions of what is possible, allow themselves to publicly express indignation, whether feigned or sincere, and manage to impose a deviant qualification of the regarded practices leading to mobilizations in several social spaces. By shifting the focus on the emerging dynamics of multisectoral mobilizations, we advocate a relational and processual approach to the emotional dimension of the scandal, that cannot be isolated from its cognitive one.

\section{INDEX}

Keywords : political scandal, indignation, mobilizations, Switzerland

Mots-clés : scandale politique, indignation, mobilisations, Suisse

\section{AUTEURS}

HERVÉ RAYNER

FABIEN THÉTAZ

BERNARD VOUTAT 